\title{
E. Sipko
}

Candidate of Engineering Sciences, Department of Information Technology Design sipko888@gmail.com.,orcid.org/0000-0003-1385-119X., Cherkassy State Technological University, Ukraine

\section{O. Kravchenko}

Candidate of Engineering Sciences, Associated Professor

Department of Information Technology

kravchenko_ov@gmail.com.,orcid.org/0000-0002-9669-2579..

Taras Shevchenko National University of Kyiv, Ukraine
A.Karapetyan
Candidate of Technical Sciences, Department of Information
Technology Design
anait.r.karapetyan@gmail.com., orcid.org/0000-0002-7412-3252.
Cherkassy State Technological University, Ukraine

\section{Zh. Plakasova}

Senior Lecturer, Department of Automated Systems Software zh.plakasova@chdtu.edu.ua., orcid.org/0000-0003-3911-2600., Cherkassy State Technological University, Ukraine

\section{Gladka}

Assistant Department of Information Systems and Technology miragladka@gmail.com., orcid.org/0000-0001-5233-2021

Taras Shevchenko National University of Kyiv, Ukraine

\section{THE SYSTEM RECOGNIZES SURFACE DEFECTS OF MARBLE SLABS BASED ON SEGMENTATION METHODS}

\begin{abstract}
A system for recognizing surface defects in marble slabs is proposed. The pattern recognition method based on segmentation methods was further developed. The algorithm of the recognition system. The article describes methods for determining damage from digital images on various hard surfaces. Research in this field is relevant for a wide range of industrial enterprises that specialize in the production of various kinds of materials: parts, marble slabs, building materials, etc. To solve this problem, it is proposed to use the k-means clustering method.

It has been experimentally established that Gaussian blurring algorithms, the Hough transform, and the Kenny algorithm are best suited for recognizing defects on the surface of a marble slab.

The developed complex method based on the theory of pattern recognition allows you to quickly identify defects and damage on the surfaces of marble slabs. On the basis of the method, a system for understanding defects is implemented in software. The main stages of the system are described in the article. The results of the analysis of the image of the surface of the marble slab on a specific example are presented.

The developed complex method based on the theory of pattern recognition allows you to quickly identify defects and damage on the surfaces of marble slabs. On the basis of the method, a system for understanding defects is implemented in software. The main stages of the system are described in the article. The results of the analysis of the image of the surface of the marble slab on a specific example are presented.
\end{abstract}


Key words: vision system, segmentation, adaptation, method, recognition, digital image.

\section{Introduction}

Among the many problems that are solved on the way to automating the production of an industrial enterprise, the creation of theoretical and experimental prerequisites for building industrial systems for processing visual information on the basis of modern information technologies is very important.

These include systems for determining objects, systems for automated control of product quality (control of geometric parameters, defect detection), systems for analyzing experimental graphs, curves, images, etc. All this allows you to get the best results in improving product quality and labor productivity by compared to the use of human resources. The greatest effect from the introduction of information technology in production should be expected from automatic image processing. Since they are directly related to production processes.

Digital image processing is a scientific industry that is rapidly developing today and is widely used in various fields of scientific and practical research. There are three groups of typical image processing algorithms that are characteristic of all vision systems: preliminary image processing; segmentation; description. The purpose of image pre-processing is the formation and further improvement of the image, its binarization and coding (in particular, obtaining a contour representation). Segmentation algorithms, as a rule, are based on two procedures: search for discontinuity in the circuit; determining the similarity of areas. An important role in the process of segmentation is played by the choice of the corresponding uniformity, which makes it possible to distinguish between the analyzed objects. Mostly used spectral and texture characteristics.

\section{Analysis of literary tribute and problem statement}

Assessment of the state of the working surface of various coatings by their digital image has become widespread [1-3]. This approach allows you to automatically analyze the surface and obtain important information about the surface and internal changes in the structure of the material, which are of interest from the point of view of its operational characteristics. This is especially true for the production of building materials.

In modern construction, the term «marble» is understood not only as marble, but also other dense transitional carbonate rocks that can be easily polished. The color scheme and pattern of the stone depends on the conditions of its origin. By decorativeness, machinability and conditions of use, it is customary to divide marble into three groups: white, gray and color. Natural blotches, streaks and color shades are a virtue, not a defect in marble. How to determine the defect of a marble slab?

Obviously, even the most attentive quality control inspector can make a mistake at the stage of checking the surface of marble. In addition, such a check may take quite a long time. This factor greatly affects the throughput of production and does not provide sufficient quality of the finished raw material precisely because of the human factor. This imposes quite strong restrictions on production in general.

Therefore, it is advisable to use information technology to save time and reduce errors in recognizing defects in marble.

There are many image processing methods that solve problems in specific fields. Thus, the problem arises of adapting or developing new algorithms that ensure the detection and classification of surface defects of marble slabs.

Such scientists as Zhuravlev Yu.I., Soifer V.A., Sergeev V.V., Ablameyko C.B., Aleksandrov V.V., Gorsky N.D., Vasin Yu. Made a great contribution to the theory of pattern recognition and image processing. G., D. Marr, R. Gonzalez, D. Forsyth, M. Sonka, W. Pratt and others. 
At the moment, there are a huge number of systems that will, to varying degrees, identify defects and damage to the hard surfaces of various materials. The most advanced of them are based on a rich database of images of reference and defective areas of various kinds. Some of the most effective in this field are ZetaScan, WebRanger, and CV-5000 series systems. However, the methods underlying them are not published in the open press, as they are commercial in nature. In addition, each of these systems has significant drawbacks.

For example, in CV-5000 and ZetaScan series systems, materials being inspected are limited in size. The WebRanger system allows you to recognize only defects of surfaces of a homogeneous type, besides mechanical, or caused by the ingress of foreign substances, bodies on these same surfaces. This complicates the solution of the problem with the recognition of defects on the surfaces of marble slabs due to the presence of a natural pattern on it.

Thus, there is a need to create, modify, combine or improve methods of analysis and image processing.

Currently, there is no general theory of image segmentation of solid surfaces of materials. It allows you to get comprehensive recommendations on the optimal choice of a segmentation method and a set of input data [4]. There are such signs of qualitative segmentation [5]:

- homogeneity of the region in terms of characteristics (primarily in color and texture);

- the difference in the values of the selected characteristics for adjacent areas of the image;

- smoothness of the boundaries of each image segment;

- a small number of «holes» in the segment.

It is known that the most effective methods of segmentation are developed for specific tasks taking into account the specifics of the image [5-7].

\section{The purpose and objectives of the study}

The aim of the study is to improve methods for determining damage from digital images on various hard surfaces.

To achieve the goal, the following tasks were set:

- analysis of the main approaches to image segmentation;

- development of a system for recognizing surface defects of marble slabs;

- verification of the system.

\section{Analysis of the main approaches to image segmentation.}

By segmentation in this work, we mean the procedures for selecting objects in the image according to given groups of indicators and their numerical values, or by similar signs. This is a very time consuming process. In general, the process is not fully algorithmized for arbitrary images. Various conditions for obtaining data, side factors - this is not a complete list of reasons, translate solutions to comparison problems in the category of extraordinary [8].

One of the most common approaches to image segmentation in the absence of a training set is based on the use of data clustering algorithms. Clustering is a fundamental tool in recognition and learning tasks and has a long and rich history of development in various scientific fields.

In a general statement, the task of clustering is to split the set of classified objects into a relatively small number of disjoint subsets (clusters). Each cluster should consist of similar objects. The objects of different clusters should differ significantly [9]. In this case, the question of determining the number of clusters in the data and the choice of a measure of similarity does not have a clear answer and, as a rule, they are selected based on the characteristics of the problem being solved [10]. 
To date, several hundred different clustering algorithms for multidimensional data are known. Many review articles and monographs on clustering have been published. A large number of publications indicates the complexity of the problem and the impossibility of creating a universal clustering algorithm [11].

Segmentation algorithm Floodfill. Using FloodFill (fill), you can select areas of uniform color. To do this, select the initial pixel and set the interval for changing the color of neighboring pixels relative to the original. The interval may be asymmetric. The algorithm will combine the pixels into one segment (filling them with one color) if they fall into the specified range. The output will be a segment filled with a certain color, and its area in pixels. Such an algorithm can be useful for filling in an area with weak color differences with a uniform background. One option for using FloodFill can be to identify damaged corners of an object.

MeanShift Segmentation Algorithm. MeanShift is a data clustering algorithm that is often used for computer vision and image processing. MeanShift groups objects with similar attributes. Pixels with similar features are combined into one segment and at the output we get an image with homogeneous areas. If you select points and intensity intensities by colors as signs, pixels of similar color and located close to each other will be combined into one segment. Accordingly, if you select a different vector of features, then the union of pixels into segments will already go along it. If the object we want to highlight consists of areas that vary greatly in color, then MeanShift will not be able to combine these regions into one, and our object will consist of several segments, but it will cope well with a uniform object in color against a motley background.

The watershed segmentation algorithm (WaterShed). It is proposed to consider the image as a certain map of the area, where the brightness values are the values of the heights relative to a certain level. If this area is filled with water, then pools form. Upon further filling with water, these pools combine. The places of association of these basins are marked as watershed lines. When the two regions begin to merge, a partition is built to prevent the unification of the regions.

Water will continue to rise until the regions are separated only by artificially constructed partitions. The algorithm can be useful if the image has a small number of local minima. In the case of a large number of them, an excessive segmentation occurs. As a result of the algorithm, we get a mask with a segmented image, where the pixels of one segment are denoted by the same tags and form a connected area.

The main disadvantage of this algorithm is the use of the preprocessing procedure for pictures with a large number of local minima (an image with a complex texture and with a large number of different colors).

The marker watershed method is one of the most effective image segmentation methods. When implementing this method, the following basic procedures are performed:

- the segmentation function is calculated. It concerns images where objects are placed in dark areas and are difficult to distinguish;

- calculation of foreground markers of images. They are calculated based on the analysis of the pixel connectivity of each object;

- calculation of background markers. They are pixels that are not parts of objects;

- modification of the segmentation function based on the location values of background markers and foreground markers;

- calculations based on a modified segmentation function.

Kenny's Algorithm. The operator to select the borders of the image. Kenny's algorithm has several advantages:

- good detection;

- correct identification of the position of the borders; 
- the only response to one border.

- The algorithm is performed in several stages:

- blurring and noise removal (for this you can use the Gaussian blur algorithm);

- search for gradients (borders are marked where the gradient acquires the greatest value. They can have different directions, so the Kenny algorithm uses four filters to determine horizontal, vertical and diagonal edges in a blurred image);

- only local maxima are marked as boundaries;

- double threshold filtering. Potential boundaries are determined by thresholds;

- tracing the ambiguity area. The resulting boundaries are determined by suppressing all edges that are not related to certain limits.

In addition to the above segmentation algorithms, there are also auxiliary algorithms. For example, to remove noise in the image.

Gaussian Blur. A method of filtering an image using the Gaussian function, which leads to blurring of the image.

This effect is widely used in graphic programs, as a rule, to reduce image noise and reduce detail.

The visual effect of this blur filtering is similar to looking at the image through a translucent screen, significantly different from the "bokeh" effect that can be obtained using an unfocused lens or an object's shadow under normal lighting.

\section{Software implementation of a system for recognizing surface defects of marble slabs}

To solve the problem of recognition of defects in marble slabs, it was decided to use the k-means clustering method. On this basis, a system for recognizing defects in marble slabs was developed and practically implemented. System

It consists of two parts: a user interface and a data processing server, which is written in Python programming language. Django web framework was used when developing the software.

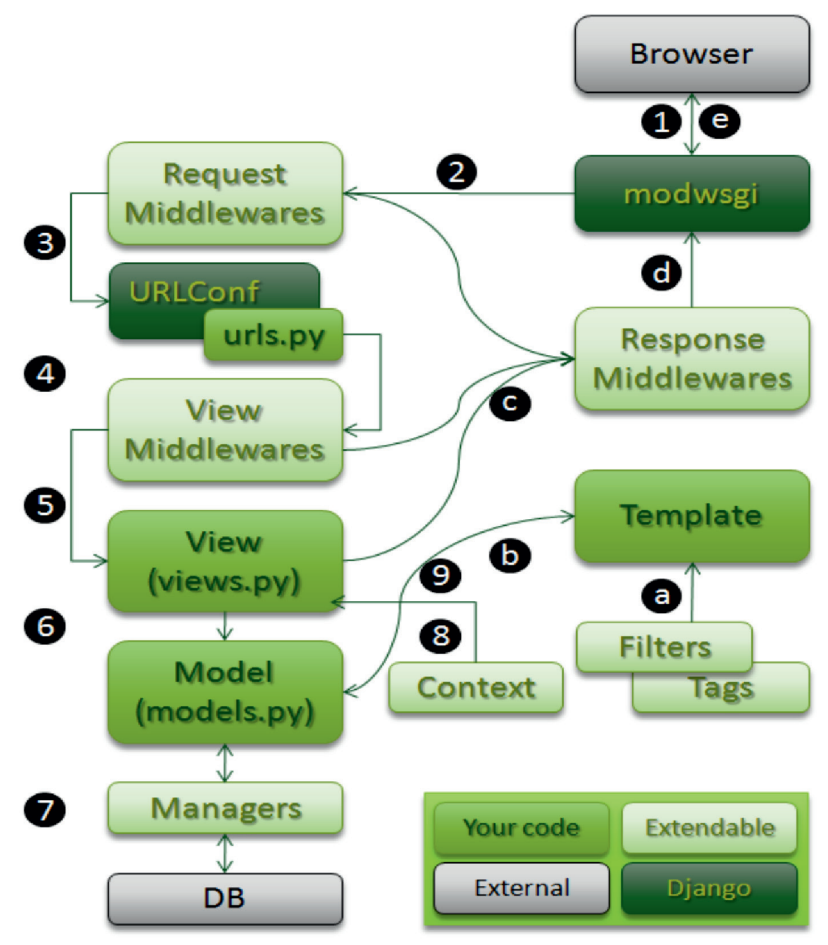

Fig. 1. Django Data Flow Chart 
Figure 1 shows a flow chart. The OpenCV library was used for image processing and analysis. The SQLite database was used to save the results of the image analysis.The Git version control system, a distributed file management and collaboration system, was used during project development.

Remote access to Git repositories is provided by the git-daemon, scheduler, SSH or HTTP server. The git-daemon TCP service is included in the Git distribution and together with SSH is the most common and reliable method of access. For Git repository operations, a SourceTree Git Client and a native Mac OS X 10.12 Sierra terminal were used. The Heroku service was used to deploy the web server.

The main function of the developed system is to display the result of defect search on the marble surface.

On the screen the system user sees:

1 Input image.

2 Original image with colored defect regions.

3 The probability that the cooker is defective.

4 Plate status.

In addition to the detailed image scan result screen, there are a number of other required screens (view images that have only been downloaded but not analyzed; images analyzed).

Figure 2 shows a diagram of a user interaction with a marble slab defect detection system.

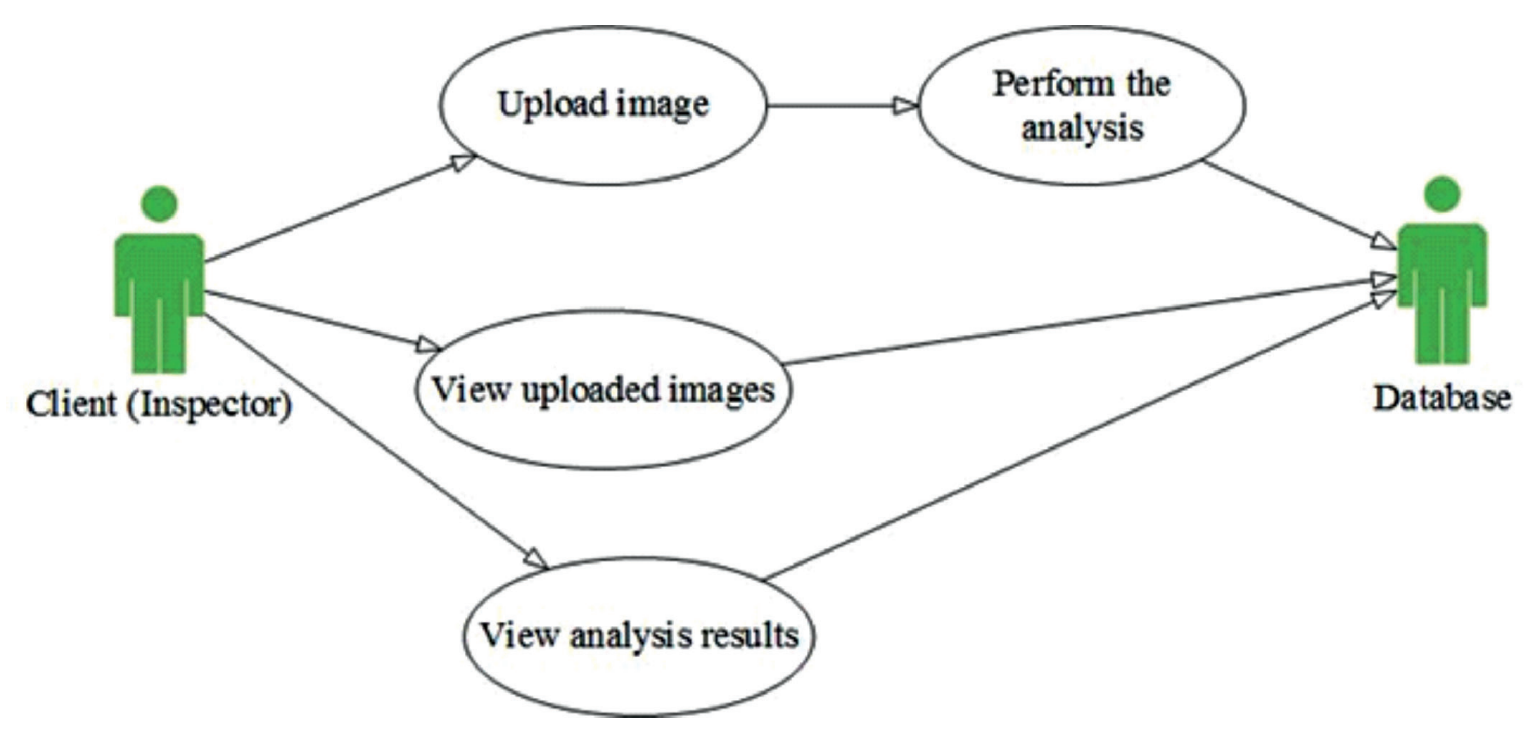

Fig. 2. Diagram of a user interaction with a marble slab defect detection system

According to the chosen method of image analysis, the work of the created system takes place in six steps. Figure 3 shows a step-by-step view of the operation of the marble slab defect recognition system. 


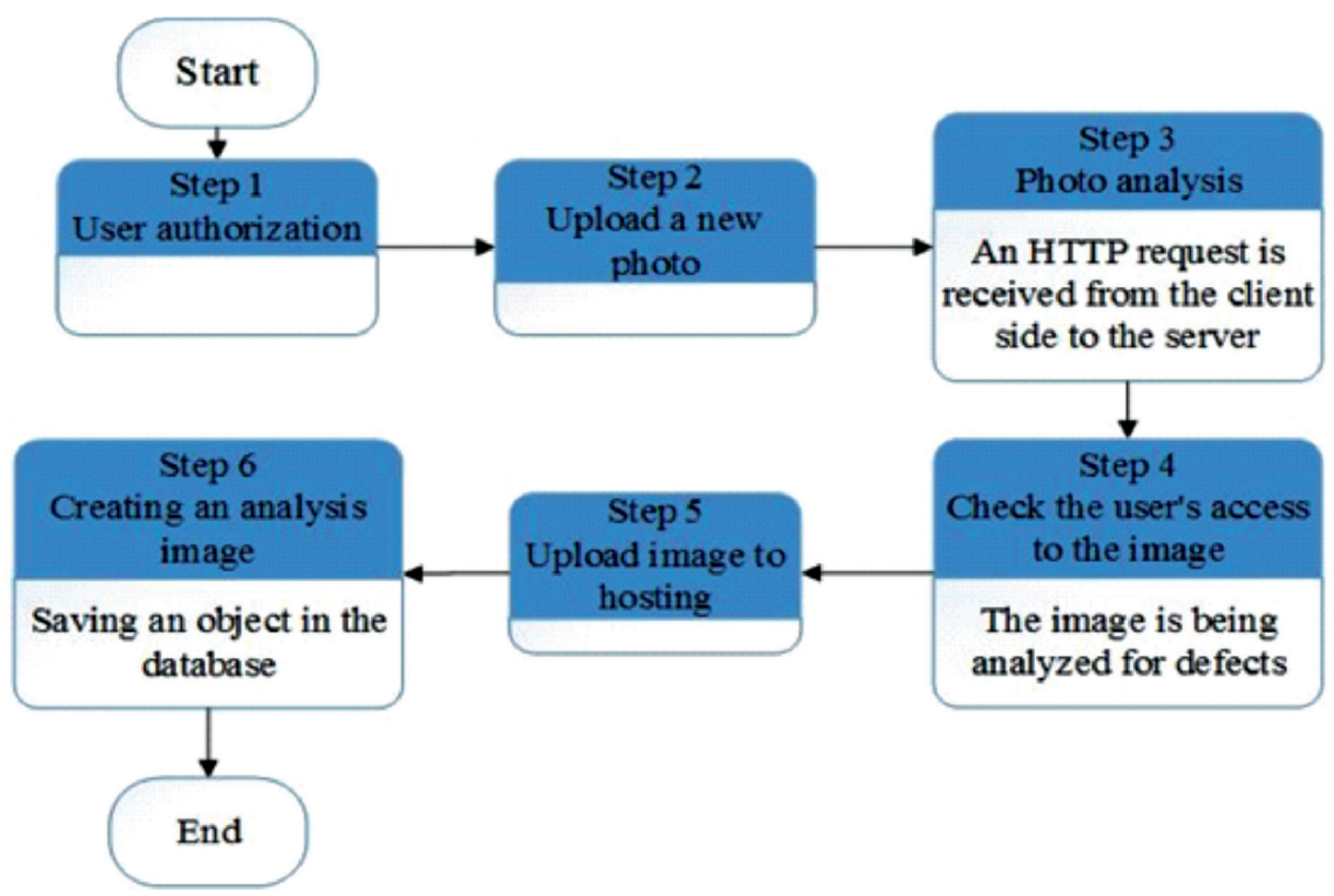

Fig. 3. Step-by-step representation of the work of a defect recognition system for marble slabs

Steps 3, 4, and 6 contain parallel processes for the marble slab defect recognition system.

\section{Research results}

To solve this problem, it is proposed to use the k-means clustering method (k-means). The main advantages of the k-means algorithm are: ease of use; speed of use; understandability and transparency of the algorithm. The disadvantages of the k-means algorithm include: the algorithm is too sensitive to outliers that can distort the average; the algorithm can work slowly on large databases. A possible solution to this problem is to use a data fetch. It has also been experimentally established that Gaussian blurring, Hough Line Transform, and Kenny's algorithms are best suited to develop a marble slab defect detection system.

Before using the detector, you should convert the image to shades of gray to reduce computational costs. This stage is characteristic of many image processing methods.

The results of the image analysis of the surface of the marble slab are shown in Figure 4.

a)

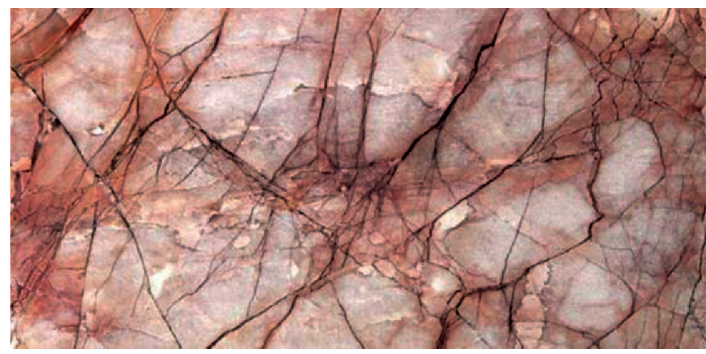

b)

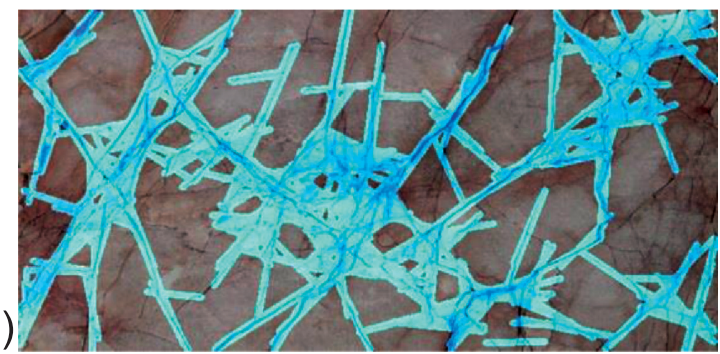

Fig. 4. Results of recognition of defects on a marble slab: $a$ - initial image, $b$ - image after defect and damage determination 


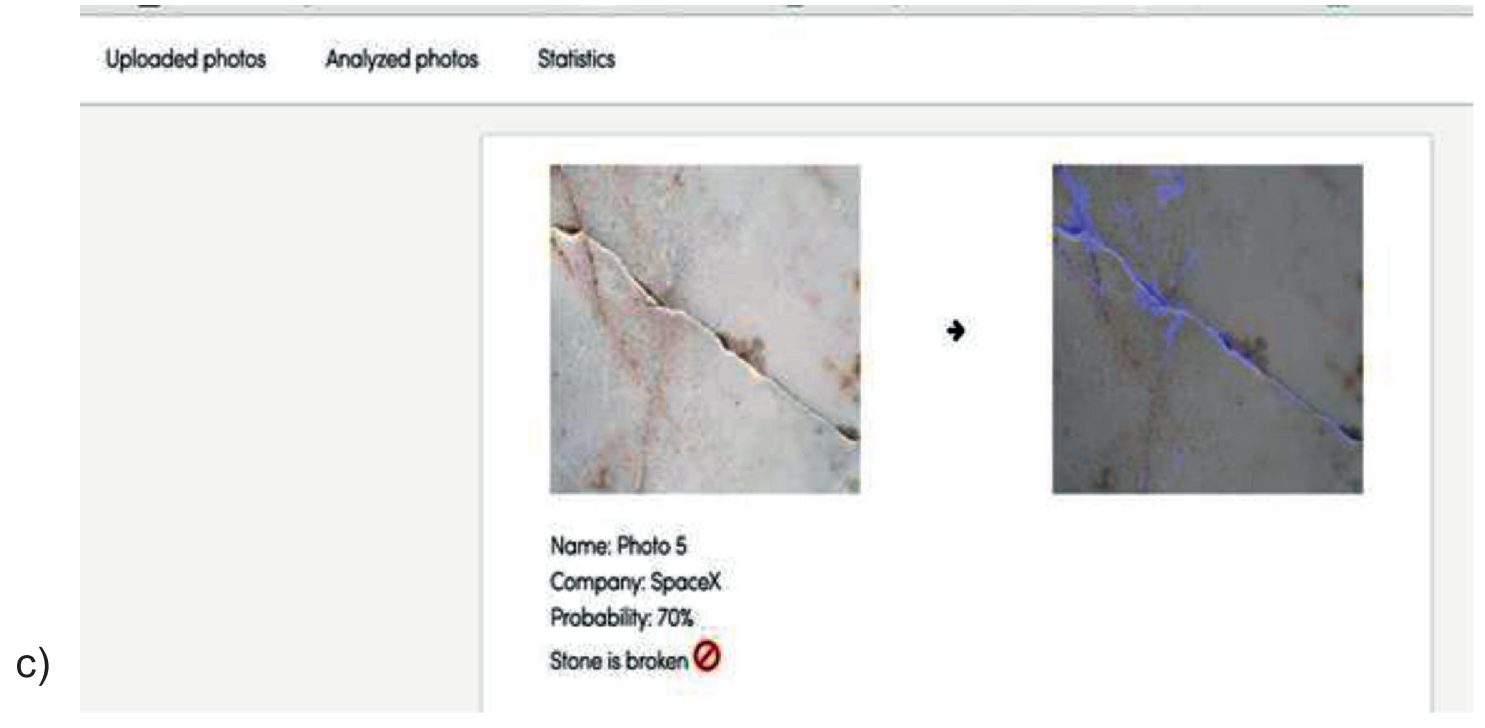

Fig. 4. Results of recognition of defects on a marble slab:

$c$ - results of recognition algorithms with determination of damage level

\section{Discussion of results}

It has been found that Gaussian blurring, Hough Line Transform, and Kenny's algorithms are best suited to develop a marble slab defect detection system [12].

Hough Line Transform a popular method for identifying any geometric shapes, if they can be represented in mathematical form. He can detect a figure, even if it is slightly broken or destroyed [13].

An analysis has been made of several widely used segmentation algorithms in order to select a marble tile slab defect analysis suitable for the task [14-15]. The success of image analysis depends on the reliability of segmentation. As a result of segmentation, areas in which pixels are combined according to selected characteristics are selected in the image. Cluster analysis collects data that contains information about a sample of objects, and then organizes the objects into relatively homogeneous groups (clusters). FloodFill algorithm is suitable for filling objects of uniform color. The task of separating a specific object from the background is well managed by the GrabCut segmentation algorithm. When using MeanShift, pixels similar in color and coordinates will be clustered [16]. This algorithm recognizes a uniform object in color against a motley background. WaterShed is suitable for images with a simple texture. To find geometric shapes in the picture, use the Hough Line Transform. Using the Kenny method, the borders in the picture are easily found.

Thus, the segmentation algorithm should be selected based on a specific task.

\section{Inference}

In the course of studying the problems of this subject area, a number of popular algorithms were found for searching for certain features in images and the most effective ones were chosen. simplification of the task of inspecting the quality of the surface of marble slabs by using the developed system will minimize the influence of the human factor on the quality of this procedure and significantly accelerate the pace of production.

A system for recognition of surface defects of marble plates based on segmentation methods was developed. the system operation was verified using pink marble slabs as an example. an image of a plate that has defects using the created system is obtained.

In the future, it is planned to take into account fuzzy logic to identify defects and damage to various materials from $3 \mathrm{~d}$ images. 
Digital image processing is a scientific industry that is rapidly developing today and is widely used in various fields of scientific and practical research. There are three groups of typical image processing algorithms that are characteristic of all vision systems: preliminary image processing; segmentation; description. The purpose of image pre-processing is the formation and further improvement of the image, its binarization and coding (in particular, obtaining a contour representation). Segmentation algorithms, as a rule, are based on two procedures: search for discontinuity in the circuit; determining the similarity of areas. An important role in the process of segmentation is played by the choice of the corresponding uniformity, which makes it possible to distinguish between the analyzed objects. Mostly used spectral and texture characteristics.

The article discusses the most popular algorithms and methods of image segmentation today, in particular, to solve the problem of recognition of defects on the surfaces of marble slabs. The main advantages and disadvantages of the considered methods are determined.

To solve this problem, it is proposed to use the k-means clustering method. It was also experimentally established that for the recognition of defects on the surface of a marble slab, Gaussian blur algorithms, the Hough transform, and the Kenny algorithm are best suited.

In the future, it is planned to continue research in this area using the methods and algorithms of fuzzy logic and fuzzy sets.

\section{References}

1. Gavil'an, M. (2011). Adaptive road crack detection system by pavement classification / M. Gavil'an, D. Balcones, O. Marcos et al.//Sensors, 10, 9628-9657; DOI 10.3390/s111009628

2. Konovalenko, I., Maruschak, Menou, P., Karuskevich, A., Ignatovich, S. (2013). A novel algorithm for damage analysis of fatigue sensor by surface deformation relief parameters. In.: Proc. of International Symposium "Operational research and applications", Marrakech, Morocco, May 08-10, 2013, p. 678-684.

3. Maruschak, P.O., Panin, S.V., Ignatovich, S.R., Zakiev, I.M., Konovalenko, I.V., Lytvynenko, I.V., and Sergeev, V.P. (2012). Influence of deformation process in material at multiple cracking and fragmentation of nanocoat ing, Theor. Appl. Fract. Mechan., vol. 57, pp. 43-48.

4. Smelyakov K., Romanenko I., Ruban I. (2010). Methods of segmentation of images of irregular-looking objects, peculiarities of their application and prospects for development. Proceedings of Kharkiv University of the Air Force. - Issue, 2 (24). - pp. 92-97.

5. BartalevS., Khovratovich T.(2011).Analizvozmozhnostei primeneniya metodov segmentatsii dlya vyyavleniya izmenenii $v$ lesakh [Analysis of the possibilities of applying satellite image segmentation techniques to detect changes in forests]. Sovremennyye problemy distantsionnogo zondirovaniya Zemli iz kosmosa - Modern problems of remote sensing of the Earth from space. (Vol. 8), (pp. 44-62). [In Russian]

6. Samoylenko D.Ye. (2004). Strukturnaya segmentatsiya izobrazheniy [Structural Image Segmentation]. // Shtuchnyy intelekt - Artificial Intelligence, 4, 521-528. [in Russian]

7. Whitey, D.J. \& Koles, Z.J. (2008). A review of Medical Image segmentation: Methods and available software. International Journal of Bioelectromagnetism (Vol. 10, 3), pp. 125-148.

8. Duda R., Khart P. (2013). Raspoznavaniye obrazov i analiz stsen [Pattern Recognition and Scene Analysis]. Moscow: Kniga po Trebovaniyu. [in Russian]

9. Jain, A.K. (2010). Data clustering: 50 years beyond K-means. Pattern recognition letters. (Vol. 31, 8), pp. 651-666.

10. Xu, R. \& Wunsch D. (2005). Survey of clustering algorithms. IEEE Transactions, Neural Networks. (Vol. 16, 3), pp. 645-678. 
11. Kashef, R. \& Kamel, M.S. (2010). Cooperative clustering. Pattern Recognition. (Vol. 43, 6), pp. 2315-2329.

12. Oliveira, H. and Correia, P.L. (2013). Automatic road crack detection and charac-terization. IEEE Trans. Intell. Transp. Syst., vol. 14, no. 1, pp. 155-168, Mar. 2013.

13. Avila, S. Begot, F. Duculty, and T. S. Nguyen. (2014). 2D imagebased road pavement crack detection by calculating minimal pathsand dynamic programming. In Proc. Int. Conf. Image Process., pp. 783-787.

14. Delling, D., Sanders, P., Schultes, D. and Wagner, D. (2009). Engineering routeplanning algorithms. In Algorithmics of Large and Complex Networks, ser. Lecture Notes in Computer Science, vol. 5515. Berlin, Germany:Springer-Verlag, pp. 117-139.

15. Belongie S., Mori G. \& Malik J. (2006). Matching with shape contexts. In Statistics and Analysis of Shapes. 105.

16. Frucci, M., Sanniti di Baja, G. (2008). From Segmentation to Binarization of Gray-level Images. Journal of Pattern Recognition Research. 3 (1): 1-13. DOI:10.13176/11.54 